\title{
LA MIGRACIÓN CIRCULAR FEMENINA MARROQUÍ EN HUELVA. IMPACTO Y CAMBIO ${ }^{1}$ THE CIRCULAR MIGRATION OF MOROCCAN WOMEN IN HUELVA. IMPACT AND CHANGE.
}

\author{
Chadia Arab \\ Investigadora del CNRS (Centro Nacional francés de Investigación Científica) \\ UMR ESO - Espaces et sociétés (6590) Carta, Université d'Angers \\ MSH-5bis, Bd Lavoisier - 49045 ANGERS Cedex \\ chadia.arab@univ-angers.fr \\ Recibido: 06/10/2010 \\ Aceptado: 22/12/2010
}

\begin{abstract}
Resumen:
El objetivo de este artículo es comprender la migración circular de las mujeres marroquís entre Marruecos y la provincia de Huelva y el impacto que esta migración produce una vez que éstas regresan a su país.

Esta migración se inscribe en un programa financiado por la Unión Europea cuyo fin es encontrar trabajadoras para recolectar la fresa de la provincia de Huelva durante algunos meses del año, al mismo tiempo que lucha contra la inmigración clandestina. Dicho programa existe desde 2007 y ha permitido a miles de mujeres viajar para trabajar algunos meses en España. Esta migración está supeditada a que estas mujeres regresen a Marruecos.

Palabras clave: migración femenina; circulación migratoria; desarrollo; Marruecos.
\end{abstract}

\section{Abstract:}

This article aims to examine the circular migration of Moroccan women between Morocco and the province of Huelva, and the effect of this migration when these women return to their country.

\footnotetext{
${ }^{1}$ Presentado en el Seminario Internacional Migraciones Circulares, el retorno como estrategia de desarrollo en los países de Origen, celebrado en Alicante, del 6 al 8 de octubre de 2010, organizado por el Instituto Interuniversitario de Desarrollo Social y Paz, con la colaboración del Ministerio de Ciencia e Innovación (Ref. CSO2009-07331-E) y de la Conselleria d'Educació de la Generalitat Valenciana (Ref. AORG/2010/124)
} 
This migration is part of a European Union funded programme, the purpose of which is to find female workers to pick strawberries in the province of Huelva for several months of the year, while also fighting against clandestine immigration. The programme was introduced in 2007 and has enabled thousands of women to travel to Spain to work for several months. This migration is subject to the condition that the women return to Morocco.

Keywords: female migration; migratory circulation; development; Morocco.

\section{INTRODUCCIÓN}

En el marco de los contratos en origen y de la migración circular, miles de mujeres marroquíes se desplazaron hasta Huelva para la recolección de la fresa entre 2006 y 2010. Se trataba de mujeres casadas, divorciadas, o incluso viudas, pero todas tenían hijos, ya que éste era un requisito imprescindible para su contratación. Esta política de mano de obra entre España y Marruecos es claramente sexista y, al mismo tiempo, permite luchar contra la migración clandestina mediante un sistema bien engrasado que está financiado por la Unión Europea (Programa AENEAS). El proyecto se denomina "Programa de Gestión Ética de la Inmigración Temporera" entre Marruecos y la provincia de Huelva. El Ayuntamiento de Cartaya, en colaboración con ANAPEC (la Agencia Nacional de Promoción del Empleo y las Competencias, equivalente al INEM en España), debe articular espacios, instrumentos y sistemas de gestión ética de esta mano de obra agrícola. Detrás de la teoría de esta gestión, hay mujeres que dejan a sus hijos y en ocasiones a sus maridos y familiares, y que circulan entre Huelva y Marruecos para trabajar tres meses al año en territorio español. Este trabajo, inscrito en el marco de una migración circular organizada, permite a algunas de ellas vivir todo el año de los ahorros que consiguen con ese trabajo, alimentar a la familia que se ha quedado en Marruecos y atender sus necesidades, comprar el mobiliario y los electrodomésticos necesarios para un hogar, y en ocasiones incluso comprarse o construirse una casa. Muy a menudo las que se encuentran en esta situación son mujeres rurales, muy pobres. Provienen de las clases más desfavorecidas y se ocupan de los trabajos más vulnerables en España.

Este trabajo pretende responder a las siguientes preguntas:

¿Quiénes se van? ¿En qué marco lo hacen? ¿A qué destinan el dinero conseguido cuando regresan a su país?

La provincia de Huelva: una región agrícola que vive gracias a la inmigración

Desde hace varias décadas, la provincia de Huelva es conocida por sus actividades turísticas y por el cultivo de la fresa. La recolección de estas fresas ha generado grandes movimientos migratorios desde hace varios años. 
Esta provincia se ha convertido en una gran región agrícola y obrera que acoge cada vez a más extranjeros, en particular a rumanos (11.434), polacos, colombianos, búlgaros y marroquís. Estos últimos ascienden a 6.256 (en 2009, fuente: INE).

Esta provincia se dio a conocer rápidamente a nivel mundial como una de las mayores productoras de fresas de Europa (Toronjo, 2007), un hecho que se debe a su modelo de gestión de la mano de obra extranjera temporera por medio de los contratos en origen. Este modelo persigue fundamentalmente luchar contra la inmigración clandestina, al tiempo que asegura la supervivencia de una agricultura intensiva. Es lo que Alain Morice (2004) denomina un "utilitarismo económico" (utilitarisme économique).

Según Dolores Redondo Toronjo (2009), la importancia de esta agricultura está teniendo un gran impacto económico y social en el mercado de trabajo local, ya que las características y el volumen de esta producción agrícola hacen necesaria la existencia de una importante mano de obra durante los meses de febrero a junio.

Una de las grandes riquezas actuales de esta región reside en la globalización económica desde la producción hasta la comercialización de la fresa en los mercados europeos. Esta región, que anteriormente era una zona de emigración, se ha convertido en la actualidad en una zona de acogida temporal de los extranjeros que trabajan algunos meses en la recolección de la fresa. En un primer momento estos extranjeros eran trabajadores portugueses que no tenían más que cruzar la frontera cercana para trabajar en Huelva. Allí se encontraban con andaluces pobres que llegaban para trabajar durante algunos meses en la recogida de la fresa. Tras el crecimiento económico que España experimentó en los años 80 y 90, los españoles fueron abandonando progresivamente los trabajos duros y penosos del sector agrícola, para buscar empleo en el sector servicios.

Desde hace diez años, han sido sustituidos por trabajadores magrebís, procedentes fundamentalmente de Marruecos, y en la actualidad por trabajadoras de los países del Este (principalmente de Polonia, Rumanía y Bulgaria).

Rápidamente, los marroquís que podían permitírselo fueron abandonando las labores agrícolas para ocupar puestos de trabajo en la construcción o en las fábricas. Los patrones agrícolas españoles debieron entonces poner remedio a esta falta de mano de obra de forma radical, y es en ese momento cuando España pone en marcha los primeros contratos en origen con mujeres de los países del Este (Rumanía, Bulgaria y Polonia). 
Puesta en marcha del "Programa de Gestión Ética de la Inmigración Temporera" Tras la entrada en la Unión Europea de Polonia en 2004, y de Rumanía y Bulgaria en 2007, se cuestionan los contratos en origen en relación con las mujeres del Este, y es en ese momento cuando se empieza a reflexionar sobre la acogida temporal de mujeres marroquís para que trabajen en la recolección de la fresa en Huelva.

El proyecto de los contratos en origen se inscribe en el marco del programa AENEAS (Programa de asistencia financiera y técnica a los terceros países en los ámbitos de migración y asilo), financiado por la Unión Europea e iniciado en 2004. El objetivo de este programa es luchar contra la inmigración clandestina y lograr una mejor gestión de los flujos migratorios (Arab, 2009).

Este programa es el marco en el que se inscribe el convenio de colaboración Cartaya-ANAPEC, que cuenta con un presupuesto de 1,5 millones de euros. El proyecto se denomina "Programa de Gestión Ética de la Inmigración Temporera" entre Marruecos y la provincia de Huelva. El Ayuntamiento de Cartaya, en colaboración con la ANAPEC, debe articular espacios, instrumentos y sistemas de gestión ética de esta mano de obra agrícola.

La Unión Europea ha señalado este sistema como una buena práctica de inmigración a través de esta migración circular de las mujeres (Arab, 2009).

El programa prevé alojar gratuitamente a estas mujeres en el seno de las cooperativas en las que trabajan. También prevé el retorno de las mujeres que no resulten adecuadas para el trabajo por varios motivos: enfermedad, lentitud, dificultades de inserción, ser fuente de conflictos en el trabajo, etc.

¿De dónde vienen estas mujeres, cuántas son y quiénes son?

El programa entra en vigor en 2007 con la llegada de 5.000 mujeres (fuente: Ayuntamiento de Cartaya). En 2008 son más de 13.000 y alcanzan las 17.000 en 2009, la cifra más alta. Las marroquís son muy apreciadas por la calidad de su trabajo y por su docilidad en el trabajo. En 2010, debido a la crisis, la cifra baja drásticamente pero, no obstante, 5.500 siguieron participando en la recolección de la fresa entre febrero y junio de 2010.

La emigración temporera de estas mujeres afecta a todo el territorio de Marruecos. No obstante, algunas regiones se ven más afectadas que otras. Por ejemplo, las agencias de la ANAPEC de las ciudades de Kenitra $\left(1.286^{2}\right)$, Mequínez (943), Fez (713), Marrakech (647) y Agadir (647) registran más de un tercio del total de solicitudes de las mujeres.

\footnotetext{
${ }^{2}$ Se trata de estadísticas de 2008, sobre aproximadamente 13.000 mujeres marroquíes. (Fuente: Ayuntamiento de Cartaya).
} 
En 2008, de las 13.380 mujeres, 6.844 están casadas, 4.368 están divorciadas y 1.427 son viudas (fuente: Ayuntamiento de Cartaya).

Todas tienen hijos, ya que se trata de un requisito previo para su contratación.

Son varios los requisitos imprescindibles para su contratación: ser mujer, ser originaria de un medio rural, tener experiencia en la agricultura, estar casada (con carta de autorización del marido), divorciada o viuda y poderlo justificar, y como requisito más importante, tener al menos un hijo menor de 18 años. Estas condiciones permiten que tengan ataduras en su país, lo que condiciona que estas mujeres regresen a su hogar junto a sus familias.

La ANAPEC es quien se encarga del proceso de contratación de las mujeres en Marruecos y de tramitar sus expedientes. Agentes de los municipios rurales ayudan a la ANAPEC a identificar a las mujeres que pueden formar parte del contingente de migrantes temporeras.

\section{EL RETORNO DE LAS MUJERES A MARRUECOS: ¿QUÉ CAMBIOS SE PRODUCEN?, ¿QUÉ IMPACTO TIENE?}

\section{¿Cómo invierten su dinero al regresar a Marruecos?}

Durante su estancia migratoria, las mujeres desarrollan estrategias para maximizar sus ahorros, gastando lo menos posible.

De este modo, algunas de ellas evitan salir, con el fin de no gastarse el dinero, se agrupan para realizar las compras cotidianas o la de la bombona de gas, y comparten sus comidas. También se llevan comida de Marruecos. Por ejemplo, llegan con varios litros de aceite de oliva, habas, judías blancas, lentejas, especias, algunos platos y vajilla que podrán utilizar en su día a día. Compran un horno para el pan, pero lo hacen entre varias. Prefieren elaborar su propio pan en lugar de comprarlo todos los días. Todas estas estrategias les permiten reducir los gastos cotidianos.

Lejos de los objetivos masculinos, a menudo individuales porque ellos migran en primer lugar para sí mismos, la mujer migrante lo hace por falta de elección, puesto que carece de otra alternativa en Marruecos. Con frecuencia, lo hace principalmente por estrategia familiar, ya que a su regreso debe responder ante toda su familia de su migración y de su éxito.

Generalmente, cuando el hombre emigra lo hace en primer lugar para responder a un proyecto migratorio personal, a un éxito personal. Por el contrario, para la mujer se trata de un proyecto colectivo. De hecho, cuando se pregunta a una mujer la razón por la que se marcha, a menudo responde que lo hace sobre todo por sus hijos y lo hace extensivo a su marido, en caso de que lo tenga, y a sus padres, a sus hermanos y hermanas, etc. 
Los requisitos establecidos para esta migración permiten a Marruecos asegurarse el retorno de estas mujeres y Marruecos tiene un especial interés en que éstas regresen, ya que su retorno garantiza la entrada de divisas. Al final de la campaña, estas mujeres vuelven con unos dos o tres mil euros (las que han permanecido en España los tres meses y han trabajado diariamente). También envían dinero a sus familias y traen muchísimos regalos cuando regresan a Marruecos.

Si lo comparamos con España, Zhara, que en Agadir trabajaba en una fábrica, ganaba 5,50 dírhams por hora mientras que en España gana 55 dírhams (unos 5,50 euros, es decir, 35 euros al día), lo que supone multiplicar por diez su salario. Además, con una hora de trabajo en España gana lo que podría ganar en una jornada de trabajo en Marruecos. Y es fundamentalmente éste el motivo por el cual estas mujeres aceptan el desarraigo, la separación (de los hijos), el miedo de no saber lo que les espera, etc. Saben que si trabajan bien pueden ganar lo que en Marruecos les habría costado ahorrar varios meses, o incluso varios años.

El primer año, la mayoría de ellas amontona regalos. Hemos podido ver que los regalos de los que estas mujeres hacen acopio son, en primer lugar, regalos para sus hijos y para el resto de la familia: mantas, alfombras, teléfonos móviles, ropa para niños, juguetes (por ejemplo, bicicletas), etc. La mayoría de ellas, y en particular aquellas que viajan por segunda vez, prefieren llevar de vuelta dinero en lugar de regalos.

En Marruecos, invierten en el hogar comprando muebles para el salón, electrodomésticos (nevera, televisión, etc.), o instalando electricidad en sus casas, las que no la tenían. Algunas ahorran y, después de retornar por segunda vez, se han podido comprar un piso pequeño o un terreno para construir una casa.

Existe un impacto económico, pero sobre todo se producen cambios sociales. En ocasiones, e incluso podríamos decir que muy a menudo, la mujer que se marcha en este marco es una mujer que ha tenido una vida difícil. Está desvalorizada en su entorno familiar y en ocasiones todo el pueblo la señala con el dedo. El ejemplo de Zhara es revelador. La migración, pero aún más las condiciones de esta migración, que hace que se marchen mujeres divorciadas o viudas con hijos, que generalmente en Marruecos están muy poco consideradas, se ha revalorizado completamente a día de hoy.

Ejemplo de Zhara, de madre soltera de la que renegaban a migrante que asume su nuevo estatus

Zhara nació en un pueblo a varios kilómetros de Juribga, a 6 km por carretera de la ciudad de Juribga. Su familia, en la que son 8 hijos, es muy pobre y ella 
vive en la casa familiar con sus hermanos y hermanas, pero también con sus tíos, primos y primas. Nunca irá a la escuela. Ninguna niña de la familia ha sido escolarizada. Zhara se casó a los 30 años, cuando ya la consideraban demasiado mayor. Su padre la casó a la fuerza con un hombre de 70 años, un inmigrante, antiguo combatiente, que de vez en cuando se marcha a Francia. Su mujer ha fallecido y decide contraer un nuevo matrimonio con Zhara. Desde los primeros días, las hijas de su marido, que no la quieren, la maltratan. Zhara sufre y ya no soporta su matrimonio, ni la vida que lleva. Al cabo de algunos meses, vuelve a casa de sus padres y su marido solicita el divorcio. Aquí la tenemos, con 30 años y divorciada. Después tendrá una aventura con un primo, que de vez en cuando va a ayudar a la familia. Zhara tiene 32 años; él, sólo 23. Se queda embarazada y en ese momento estalla el drama familiar.

Nadie la cree, la toman por una prostituta, y sus propios hermanos y su padre la echan de casa. Su tío la acoge y ella realizará algunos pequeños trabajos domésticos a domicilio para alimentarse. Da a luz y se convierte en lo que denominamos una madre soltera. Está sola con su hijo, y unos familiares lejanos la acogen en su vivienda, mientras realiza algunos trabajos domésticos en casa de las vecinas para alimentar a su nueva familia.

Un funcionario del municipio de Juribga es el primero que le habla de marcharse a España. Le da miedo pero, al mismo tiempo, se dice a ella misma que ya no tiene nada que perder, que su vida ya es difícil con su familia más cercana que sigue renegando de ella. Pide ayuda a este funcionario, quien le explica los documentos que se necesitan para su expediente en ANAPEC. Va hasta Beni Mellal, a la ANAPEC, sin casi creérselo, y cumple todos los requisitos establecidos: mujer divorciada con un hijo. Le cuesta creer que sea cierto, ella que nunca ha salido de su casa, de su campo, que no sabe ni leer ni escribir...

Solicita el documento de identidad y después el pasaporte, y acude a la reunión de información en Mohammedia. Empieza para ella una auténtica aventura. La contratan para trabajar en la recolección de fresas en Huelva. Zhara debe organizarse, vuelve a ver a su madre y la deja al cuidado de su hijo. El niño, al que llamaban "el bastardo" se ha convertido, desde que Zhara anunció que se marchaba a Europa, en el niño "bendito", el niño que permite la migración: el niño de la "pesadilla" pasa a ser el niño que puede hacer que los sueños se cumplan. La mujer a la que habían echado de casa debido al niño es aceptada, la reintroducen en el círculo familiar y recupera su lugar en el seno de la familia. Zhara se marcha a España en 2008 para tres meses, y en 2009 vuelve a viajar para dos meses. Cada vez que regresa, organiza una sadaka (fiesta familiar y religiosa) e invita a toda su familia. Actualmente se la considera de forma diferente. Invierte en la casa y todos los miembros de la familia, que fueron responsables de su expulsión de la casa familiar, miman a su hijo. 
La migración, pero aún más las condiciones de esta migración que hace que se marchen mujeres divorciadas o viudas con hijos, que generalmente en Marruecos están muy poco consideradas, ha revalorizado completamente a estas mujeres a día de hoy.

Desde su migración, Zhara se ha metamorfoseado. Además de mejorar las condiciones de vida de su familia, ahora tiene un papel y un estatus en su ámbito familiar que no tenía anteriormente. Su voz cuenta. Se siente más libre de sus actos. A día de hoy, por ejemplo, va a trabajar incluso al margen de la temporada de fresas en España, en Marruecos. Recuerda que antes no habría podido ni siquiera pensar en trabajar en la agricultura. Hoy, a Zhara le resulta imposible estar sin hacer nada. En cuanto regresa de España, se prepara para ir a cualquier sitio de Marruecos en el que pueda trabajar. Por ejemplo, ha trabajado en la cosecha de olivas en Beni Mellal. También fue a trabajar en la recolección de manzanas en Ait Buguemez. Según las últimas noticias que tenemos de ella, quería ir a trabajar a las fábricas de Agadir.

Esta migración le ha hecho ganar confianza, reconocimiento por parte de los demás, que en la actualidad la ven de forma diferente. Su vida ha cambiado, es consciente de ello, y quiere seguir siendo parte actora de ese cambio que para Zhara se pone de manifiesto a todos los niveles.

Por tanto, las relaciones sociales dentro de la esfera familiar evolucionan gracias a la migración de la mujer joven. Si anteriormente la criticaban, e incluso su familia renegaba de ella, hoy en día su entorno familiar la admira y la pone como ejemplo de éxito familiar.

\section{CONCLUSIÓN}

Este singular Programa de Gestión Ética de la Inmigración Temporera entre Marruecos (ANAPEC) y la provincia de Huelva (Ayuntamiento de Cartaya), ha permitido a miles de mujeres marroquís desplazarse hasta España para trabajar durante unos meses en la recolección de la fresa. Sin duda alguna, estas mujeres volvieron transformadas por esta migración circular, inesperada e imprevista, puesto que antes de que fueran a buscarlas, estas mujeres nunca habían pensado migrar. Fueron elegidas porque quienes las contratan cuentan teóricamente con que regresen al terminar el trabajo. Ser mujer y tener hijos son los dos requisitos principales para esta migración: tener ataduras, raíces, que permitan, que obliguen, que impongan a estas mujeres el retorno.

A menudo, estas mujeres comparten unas características que las definen: frágiles, precarias, pobres, rurales. Están marcadas por vidas difíciles (divorcio, fallecimiento del marido, obligación de ir a trabajar...), en oca- 
siones por vidas múltiples (antes de los 35 años, han trabajado como criadas, las han maltratado, han estado marcadas por la pobreza, se han casado y divorciado, se han prostituido, etc.). Para la mayoría de ellas, la migración ha resultado beneficiosa para sus condiciones de vida y para su ascenso social. Esta experiencia las ha cambiado para siempre, en lo más profundo de ellas mismas.

El primer elemento de cambio es, en primer lugar, de tipo financiero y económico. Permite mejorar rápidamente las condiciones de vida, aunque de forma puntual y temporal. Mientras las mujeres se sigan marchando, podrán seguir enviando dinero a sus familias que se han quedado en Marruecos. Pero, cuando ya no puedan marcharse, ¿qué habrán ganado? Este asunto ya se ha planteado este año con la crisis.

El segundo elemento es la imagen que tienen de ellas mismas y que transmiten a los demás. Todas afirman que han ganado en seguridad, en sentido de la responsabilidad, en autoridad sobre ellas mismas y sobre los demás, en sentido de la economía. España, en cierta forma, las ha liberado. Liberado de ellas mismas y de los impedimentos que se impusieron, pero liberado también de la tutela masculina. Cruzar la frontera les ha permitido transgredir las reglas que les imponían o que ellas mismas se habían impuesto.

Las mujeres casadas se emancipan menos que las divorciadas o viudas. Las mujeres casadas retoman, en cuanto están en España, los roles llamados femeninos que asumían antes de su partida. Las otras están mucho más emancipadas. Algunas, que no habían salido nunca del pueblo sin que las acompañase un hombre, recorren hoy Marruecos sin dificultad, trabajan en una región distinta de aquella de la que proceden, son capaces de realizar trámites administrativos que antes dejaban en manos de los hombres, pueden plantar cara a los hombres y hablar libremente con ellos.

Pero la causa principal del papel casi masculino que asumen estas mujeres que se convierten en auténticas cabezas de familia es que tienen hijos a su cargo. L. Oso subraya que:

cette perspective d'analyse permet de plus d'approfondir, à partir de la sociologie du genre, la redéfinition des rôles sexuels, puisque la femme immigrée avec des personnes à charge assume le rôle de chef de famille, rôle traditionnellement réservé, dans le schéma patriarcal, à l'homme. (Oso, 2000: 130)

(esta perspectiva de análisis permite también profundizar, a partir de la sociología de género, en la redefinición de los roles sexuales, ya que la mujer que emigra teniendo personas a su cargo asume el papel de cabeza de familia, un rol que en el esquema patriarcal está tradicionalmente reservado al hombre.) $)^{3}$

${ }^{3}$ Nota: traducción realizada por el traductor del artículo. 


\section{BIBLIOGRAFÍA}

ARAB, C. (2007). "Le hrague ou comment les Marocains brûlent les frontières ?". Revue Hommes et Migration $N^{\circ} 1266$, monográfico dedicado al tema Les nouvelles figures de l'immigration en France et Méditerranée, marzo-abril 2007, 82-94.

ARAB, C. (2009). "Les Marocaines à Huelva sous 'contrat en origine': partir pour mieux revenir", Migrations Société, n¹25, 175-190.

ARAB, C. y SEMPERE SOUVANNAVONG, J. D. (2009). "Les jeunes harragas maghrébins se dirigeant vers l'Espagne: des rêveurs aux "brûleurs de frontières", Migrations société, numéro 125.

ARAB, C. (2009). Les Ait Ayad. La circulation migratoire des Marocains entre la France, l'Espagne et l'Italie. Géographie sociale. PUR, Presses universitaires de Rennes.

HELliO, E. (2009). "Importer des femmes pour exporter des fraises (Huelva)", Études rurales 2008/2, 182, 185-200.

MORICE, A. (2004). "Le travail sans le travailleur", Plein Droit, monográfico Immigrés, mode d'emploi, $n^{\circ} 61$, junio de 2004. Texto íntegro disponible en: http://www.gisti. org/doc/plein-droit/61/travailleurs.html

OSO, L. (2000). Limmigration en Espagne des chefs de famille. En J. Falquet, A. Goldberg-Salinasd, C. Zaidman, Cahiers du CEDREF, 91-144.

OSO CASAS, L. (2005). "La réussite paradoxale des bonnes espagnoles de Paris. Stratégies de mobilité sociale et trajectoires biographique". Femmes, genre, migration et mobilités, Revue européenne des migrations internationales, vol. $21-n^{\circ} 1$.

REDONDO TORONJO, D. (2007). Nouvelle réalité d'un vieux problème social? L'immigration dans les marchés de travail locaux. Le cas de Huelva (Espagne). En Coloquio Les migrations de l'Est vers l'Ouest. Entre mobilité et installation. Université Libre de Belgique (Octubre 2004).

REDONDO TORONJO, D. (2009). "Les 'contrats en origine' dans la production intensive des fraises à Huelva", Études rurales 2008/2, 182, 169-184. 